S. M. Mazhar, Department of Mathematics, Kuwait University, P.O. Box 5969, 13060 Safat, Kuwait

\title{
SOME REMARKS ON ABSOLUTE SUMMABILITY METHODS
}

\begin{abstract}
This note deals with certain recent results concerning summability $\left|\bar{N}, p_{n}\right|_{k}$.
\end{abstract}

1. Let $\sum a_{n}$ be a given infinite series with $\left\{s_{n}\right\}$ as the sequence of its $n$-th partial sums. Let $\sigma_{n}^{\alpha}$ and $t_{n}^{\alpha}$ denote the $n$-th $(C, \alpha)$ means of the sequences $\left\{s_{n}\right\}$ and $\left\{n a_{n}\right\}$ respectively. We denote by $\left\{p_{n}\right\}$ a sequence of positive constants such that $P_{n}=p_{0}+p_{1}+\cdots+p_{n} \rightarrow \infty$ as $n \rightarrow \infty$. The series $\sum a_{n}$ is said to be summable $|C, \alpha|_{k}, \alpha>-1, k \geq 1[8]$ if

$$
\sum_{1}^{\infty} n^{k-1}\left|\Delta \sigma_{n-1}^{\alpha}\right|^{k}=\sum_{1}^{\infty} \frac{\left|t_{n}^{\alpha}\right|^{k}}{n}<\infty .
$$

It is said to be summable $\left|\bar{N}, p_{n}\right|_{k}, k \geq 1[1]$ if

$$
\sum_{1}^{\infty}\left(\frac{P_{n}}{p_{n}}\right)^{k-1}\left|\Delta T_{n-1}\right|^{k}<\infty
$$

where

$$
T_{n}=\frac{1}{P_{n}} \sum_{k=0}^{n} p_{k} s_{k}
$$

The series $\sum a_{n}$ is said to be summable $\left|R, p_{n}\right|_{k}, k \geq 1[11]$ if

$$
\sum_{1}^{\infty} n^{k-1}\left|\Delta T_{n-1}\right|^{k}<\infty
$$

Key Words: Absolute Cesáro summability, summability $\left|\bar{N}, p_{n}\right|_{k}$, summability $\left|R, p_{n}\right|_{k}$

Mathematical Reviews subject classification: 40F05, 40D25

Received by the editors March 11, 1996

*This research was supported by Kuwait University Research Administration Project No. SM-139 
Obviously $\left|\bar{N}, p_{n}\right|_{1}=\left|R, p_{n}\right|_{1}=\left|\bar{N}, p_{n}\right|$. Also if $P_{n}=O\left(n p_{n}\right)$ then

$$
\begin{gathered}
\left|R, p_{n}\right|_{k} \Rightarrow\left|\bar{N}, p_{n}\right|_{k} \\
\text { and if } n p_{n}=O\left(P_{n}\right) \text {, then }\left|\bar{N}, p_{n}\right|_{k} \Rightarrow\left|R, p_{n}\right|_{k} .
\end{gathered}
$$

Thus if

$$
n p_{n} \asymp P_{n},
$$

then $\left|\bar{N}, p_{n}\right|_{k} \Longleftrightarrow\left|R, p_{n}\right|_{k}$.

For $p_{n}=1$ we have $|\bar{N}, 1|_{k}=|R, 1|_{k}=|C, 1|_{k}$ while for $p_{n}=1 /(n+1)$ we find from (1.5) that $|\bar{N}, 1 /(n+1)|_{k} \Rightarrow|R, 1 /(n+1)|_{k}$ but its converse is not true as can be seen by taking $\Delta T_{n-1}=n^{-1}[\log (n+1)]^{-1}$.

It is well known [7] that $|C, 1| \Rightarrow|R, \log n, 1|$ but the converse need not be true. Also it is known that

$$
|R, \log n, 1| \Longleftrightarrow\left|\bar{N}, \frac{1}{n+1}\right|=\left|R, \frac{1}{n+1}\right|_{1} .
$$

Concerning summability $|C, \alpha|$, Kogbetliantz [9] had proved that $|C, \alpha| \Rightarrow$ $|C, \beta|, \beta \geq \alpha, \alpha>-1$. The corresponding extension to summability $|C, \alpha|_{k}$ was proved by Flett [8] who has shown that $|C, \alpha|_{k} \Rightarrow|C, \beta|_{k}, k \geq 1, \beta \geq \alpha$, $\alpha>-1$. Consequently in view of (1.7) we should expect either

$$
|C, 1|_{k} \Rightarrow\left|\bar{N}, \frac{1}{n+1}\right|_{k}
$$

or

$$
|C, 1|_{k} \Rightarrow\left|R, \frac{1}{n+1}\right|_{k}
$$

Concerning inclusion relations between summability $|C, 1|_{k}$ and summability $\left|\bar{N}, p_{n}\right|_{k}$ the following results are known.

Under the condition (1.6)

$$
|C, 1|_{k} \Rightarrow\left|\bar{N}, p_{n}\right|_{k} \quad[2]
$$

and

$$
\left|\bar{N}, p_{n}\right|_{k} \Rightarrow|C, 1|_{k} \quad[3]
$$

Consequently under the condition (1.6)

$$
\left|\bar{N}, p_{n}\right|_{k} \Leftrightarrow|C, 1|_{k}
$$

For the necessity part we have the following [11].

$$
\text { If }|C, 1|_{k} \Rightarrow\left|\bar{N}, p_{n}\right|_{k} \text {, then } n p_{n}=O\left(P_{n}\right) \text {, and }
$$




$$
\text { if }\left|\bar{N}, p_{n}\right|_{k} \Rightarrow|C, 1|_{k} \text {, then } P_{n}=O\left(n p_{n}\right) .
$$

It can be easily shown that $\left\{(-1)^{n-1}\right\}$ is summable $|R, 1 /(n+1)|_{k}$ but not summable $|\bar{N}, 1 /(n+1)|_{k}$ or $|C, 1|_{k}$. In view of the relation $|\bar{N}, 1 /(n+1)|_{k} \Rightarrow$ $|R, 1 /(n+1)|_{k}$ we should expect (1.9) to hold true. Writing

$$
\begin{gathered}
\sigma_{n}=\frac{1}{n+1} \sum_{\nu=0}^{n} s_{\nu}, \quad T_{n}=\frac{1}{P_{n}} \sum_{\nu=0}^{n} \frac{s_{\nu}}{\nu+1} \text { and } \\
P_{n}=1+\frac{1}{2}+\cdots+\frac{1}{n+1}
\end{gathered}
$$

we have

$$
\begin{aligned}
\Delta T_{n} & =\left(\frac{1}{P_{n}}-\frac{1}{P_{n+1}}\right) \sum_{0}^{n} \frac{s_{\nu}}{\nu+1}-\frac{S_{n+1}}{(n+2) P_{n+1}} \\
& =\frac{p_{n+1}}{P_{n} P_{n+1}} \sum_{\nu=0}^{n}\left(P_{\nu}-1\right) \Delta \sigma_{\nu-1}+\frac{\Delta \sigma_{n}}{P_{n+1}}
\end{aligned}
$$

so that

$$
\begin{gathered}
\sum_{1}^{\infty} n^{k-1}\left|\Delta T_{n}\right|^{k} \leq \\
C \sum_{n=1}^{\infty} \frac{n^{k-1}}{\left(n \log ^{2}(n+1)\right)^{k}}\left(\sum_{\nu=0}^{n} P_{\nu}\left|\Delta \sigma_{\nu-1}\right|\right)^{k}+C \sum_{n=1}^{\infty} \frac{n^{k-1}\left|\Delta \sigma_{n}\right|^{k}}{(\log (n+1))^{k}} \\
=O(1) \sum_{n=1}^{\infty} \frac{1}{n \log ^{2 k}(n+1)} \sum_{\nu=0}^{n} P_{\nu}(\nu+1)^{k-1}\left|\Delta \sigma_{\nu-1}\right|^{k}\left(\sum_{\nu=0}^{n} \frac{P_{\nu}}{\nu+1}\right)^{k-1} \\
+O\left(\sum_{1}^{\infty} n^{k-1}\left|\Delta \sigma_{n}\right|^{k}\right)=O(1) \sum_{n=1}^{\infty} \frac{1}{n \log ^{2}(n+1)} \sum_{\nu=1}^{n} \nu^{k-1} \log (\nu+1)\left|\Delta \sigma_{\nu-1}\right|^{k} \\
+O(1)=O(1) \sum_{\nu=1}^{\infty} \nu^{k-1} \log (\nu+1)\left|\Delta \sigma_{\nu-1}\right|^{k} \sum_{n=\nu}^{\infty} \frac{1}{n \log ^{2}(n+1)}+O(1) \\
=O(1) \sum_{\nu=1}^{\infty} \nu^{k-1}\left|\Delta \sigma_{\nu-1}\right|^{k}+O(1)=O(1)
\end{gathered}
$$

Thus $|C, 1|_{k} \Rightarrow|R, 1 /(n+1)|_{k}, k \geq 1$, whereas $|C, 1|_{k} \nRightarrow|\bar{N}, 1 /(n+1)|_{k}$ as it is evident from the following special case of a result of Sarigol [12]:

$|C, 1|_{k} \Rightarrow\left|\bar{N}, p_{n}\right|_{k}$ iff 
(i) $n p_{n}=O\left(P_{n}\right)$

(ii) $\sum_{1}^{m}\left|(\nu+1) \Delta\left(P_{\nu-1}\right)+P_{\nu}\right|^{k^{\prime}} / \nu+1=O\left(P_{m}^{k^{\prime}}\right)$.

Here (ii) does not hold if $p_{n}=1 /(n+1)$.

This shows that extension of the summability $\left|\bar{N}, p_{n}\right|$ to index $k$ in the sense of (1.3) is more appropriate.

2. Bor and Thorpe in [5] gave a generalization of the result (1.12) in following way. Suppose $\left\{p_{n}\right\}$ and $\left\{q_{n}\right\}$ are two sequences of positive constants such that $P_{n}=p_{0}+p_{1}+\cdots+p_{n} \rightarrow \infty$ and $Q_{n}=q_{o}+q_{1}+\cdots+q_{n} \rightarrow \infty$ as $n \rightarrow \infty$. If

$$
\frac{p_{n}}{P_{n}} \asymp \frac{q_{n}}{Q_{n}}
$$

then

$$
\left|\bar{N}, p_{n}\right|_{k} \Rightarrow\left|\bar{N}, q_{n}\right|_{k}, \quad k \geq 1
$$

and so

$$
\left|\bar{N}, q_{n}\right|_{k} \Rightarrow\left|\bar{N}, p_{n}\right|_{k}, \quad k \geq 1
$$

Thus under (2.1)

$$
\left|\bar{N}, p_{n}\right|_{k} \Leftrightarrow\left|\bar{N}, q_{n}\right|_{k}, \quad k \geq 1 .
$$

For the necessity part Bor and Thorpe [6] showed that if $\left|\bar{N}, p_{n}\right|_{k} \Rightarrow\left|\bar{N}, q_{n}\right|_{k}$, then

$$
\frac{q_{n}}{Q_{n}}=O\left(\frac{p_{n}}{P_{n}}\right) .
$$

Consequently if $\left|\bar{N}, q_{n}\right|_{k} \Rightarrow\left|\bar{N}, p_{n}\right|_{k}$, then

$$
\frac{p_{n}}{P_{n}}=O\left(\frac{q_{n}}{Q_{n}}\right)
$$

It is clear that if (2.6) holds then

$$
\sum_{1}^{n} \frac{p_{\nu} Q_{\nu}}{P_{\nu}}=O\left(\sum_{1}^{n} q_{\nu}\right)=O\left(Q_{n}\right) .
$$

We find that it is possible to relax the condition (2.1). We will show that if (2.5) and (2.7) hold then $\left|\bar{N}, p_{n}\right|_{k} \Rightarrow\left|\bar{N}, q_{n}\right|_{k}, k \geq 1$. This generalizes a result of Bor and Thorpe [5]. Writing

$$
t_{n}=\frac{1}{P_{n}} \sum_{\nu=0}^{n} p_{\nu} s_{\nu} \text { and } T_{n}=\frac{1}{Q_{n}} \sum_{\nu=0}^{n} q_{\nu} s_{\nu},
$$


then as shown by Bor and Thorpe [5] (pp. 147-148),

$$
\begin{aligned}
\Delta T_{n-1}= & \frac{q_{n} P_{n} \Delta t_{n-1}}{p_{n} Q_{n}}-\frac{q_{n}}{Q_{n} Q_{n-1}} \sum_{\nu=1}^{n-1} \frac{P_{\nu}}{p_{\nu}} q_{\nu} \Delta t_{\nu-1} \\
& +\frac{q_{n}}{Q_{n} Q_{n-1}} \sum_{\nu=1}^{n-1} Q_{\nu} \Delta t_{\nu-1} \\
= & T_{n, 1}+T_{n, 2}+T_{n, 3}, \text { say . }
\end{aligned}
$$

In view of Minkowski's inequality, to prove that

$$
\sum_{1}^{\infty}\left(\frac{Q_{n}}{q_{n}}\right)^{k-1}\left|\Delta T_{n-1}\right|^{k}<\infty
$$

it is enough to prove that

$$
\sum_{1}^{\infty}\left(\frac{Q_{n}}{q_{n}}\right)^{k-1}\left|\Delta T_{n, r}\right|^{k}<\infty, \quad r=1,2,3 .
$$

The proof of (2.8) for $r=1,2$ is the same as in [5], since these involve the condition (2.5) only. To prove (2.8) for $r=3$ we have in view of $(2.7)$

$$
\begin{gathered}
\sum_{1}^{\infty}\left(\frac{Q_{n}}{q_{n}}\right)^{k-1}\left|T_{n, 3}\right|^{k}=\sum_{1}^{\infty}\left(\frac{Q_{n}}{q_{n}}\right)^{k-1}\left(\frac{q_{n}}{Q_{n} Q_{n-1}}\right)^{k}\left|\sum_{\nu=1}^{n-1} Q_{\nu} \Delta t_{\nu-1}\right|^{k} \\
\leq \sum_{1}^{\infty} \frac{q_{n}}{Q_{n} Q_{n-1}^{k}}\left(\sum_{\nu=1}^{n-1}\left(\frac{P_{\nu}}{p_{\nu}}\right)^{\frac{1}{k^{\prime}}}\left(\frac{p_{\nu}}{P_{\nu}}\right)^{\frac{1}{k^{\prime}}} Q_{\nu}^{\frac{1}{k^{\prime}}} \cdot Q_{\nu}^{\frac{1}{k}}\left|\Delta t_{\nu-1}\right|\right)^{k} \\
\leq \sum_{1}^{\infty} \frac{q_{n}}{Q_{n} Q_{n-1}^{k}}\left(\sum_{\nu=1}^{n-1}\left(\frac{P_{\nu}}{p_{\nu}}\right)^{k-1} Q_{\nu}\left|\Delta t_{\nu-1}\right|^{k}\right)\left(\sum_{\nu=1}^{n-1} \frac{p_{\nu}}{P_{\nu}} Q_{\nu}\right)^{k-1} \\
=O(1) \sum_{1}^{\infty} \frac{q_{n}}{Q_{n} Q_{n-1}^{k}} Q_{n-1}^{k-1} \cdot \sum_{\nu=1}^{n-1}\left(\frac{P_{\nu}}{p_{\nu}}\right)^{k-1} Q_{\nu}\left|\Delta t_{\nu-1}\right|^{k} \\
=O(1) \sum_{\nu=1}^{\infty}\left(\frac{P_{\nu}}{p_{\nu}}\right)^{k-1} Q_{\nu}\left|\Delta t_{\nu-1}\right|^{k} \sum_{n=\nu+1}^{\infty} \frac{q_{n}}{Q_{n} Q_{n-1}} \\
=O(1) \sum_{\nu=1}^{\infty}\left(\frac{P_{\nu}}{p_{\nu}}\right)^{k-1}\left|\Delta t_{\nu-1}\right|^{k}<\infty^{\infty}
\end{gathered}
$$


This completes the proof of our result.

Interchanging $p_{n}, P_{n}$ with $q_{n}, Q_{n}$ respectively we conclude that if

$$
\frac{p_{n}}{P_{n}}=O\left(\frac{q_{n}}{Q_{n}}\right) \text { and } \sum_{1}^{n} \frac{q_{\nu} P_{\nu}}{Q_{\nu}}=O\left(P_{n}\right)
$$

then $\left|\bar{N}, q_{n}\right|_{k} \Rightarrow\left|\bar{N}, p_{n}\right|_{k}, k \geq 1$. Taking $p_{n}=1$ we get generalizations of (1.10) and (1.11).

Remark. It may be observed that (2.5) is a necessary condition while (2.7) is only sufficient. A special case of Sarigol's result [12] states:

Necessary and sufficient conditions for $\left|\bar{N}, p_{n}\right|_{k} \Rightarrow\left|\bar{N}, q_{n}\right|_{k}$, are

$$
\frac{q_{n}}{Q_{n}}=O\left(\frac{p_{n}}{P_{n}}\right)
$$

$$
\sum_{1}^{m}\left|\frac{P_{\nu}}{p_{\nu}}\left(\Delta Q_{\nu-1}\right)+Q_{\nu}\right|^{k^{\prime}} \frac{p_{\nu}}{P_{\nu}}=O\left(Q_{m}^{k^{\prime}}\right)
$$

where $1 / k+1 / k^{\prime}=1$.

Here (I) is the same as (2.5). Using (2.5) and (2.7) we have

$$
\begin{gathered}
\sum_{1}^{m}\left|\frac{P_{\nu}}{p_{\nu}}\left(-q_{\nu}\right)+Q_{\nu}\right|^{k^{\prime}} \frac{p_{\nu}}{P_{\nu}} \\
=O(1) \sum_{1}^{m} Q_{\nu}^{k^{\prime}} \frac{p_{\nu}}{P_{\nu}}=O\left(Q_{m}^{k^{\prime}-1}\right) \sum_{1}^{m} \frac{Q_{\nu} p_{\nu}}{P_{\nu}}=O\left(Q_{m}^{k^{\prime}}\right) .
\end{gathered}
$$

Hence $(2.5)$ and $(2.7) \Rightarrow(2.10)$.

Thus our result can be deduced from Sarigol's result. However we include the proof of our result as the condition (2.7) is simpler than (2.10) and our proof is not based on functional analysis method as is the case with that of Sarigol.

3. With a view to generalize the result (1.11), Bor [4] recently proved that if $n p_{n} \asymp P_{n}$ and

$$
\sum_{1}^{\infty}\left(\frac{P_{n}}{p_{n}}\right)^{(2-\alpha) k-1}\left|\Delta T_{n-1}\right|^{k}<\infty \quad 0<\alpha \leq 1, \quad k \geq 1,
$$


where $T_{n}$ denotes the $\left(\bar{N}, p_{n}\right)$ mean of a series $\sum a_{n}$, then the series $\sum a_{n}$ is summable $|C, \alpha|_{k}$.

It is clear that if $n p_{n} \asymp P_{n}$ then (3.1) is equivalent to the condition

$$
\sum_{1}^{\infty} n^{(2-\alpha) k-1}\left|\Delta T_{n-1}\right|^{k}<\infty .
$$

Thus Bor's result can be stated as: If $n p_{n} \asymp P_{n}$ and (3.2) holds then $\sum a_{n}$ is summable $|C, \alpha|_{k}, 0<\alpha \leq 1, k \geq 1$. Analyzing the proof of Bor we observe that the following result holds: If $P_{n}=O\left(n p_{n}\right)$ and (3.2) holds, then $\sum a_{n}$ is summable $|C, \alpha|_{k}, 0<\alpha \leq 1, k \geq 1$. This shows that the condition $n p_{n}=O\left(P_{n}\right)$ in Bor's result is superfluous.

\section{References}

[1] H. Bor, On $\left|\bar{N}, p_{n}\right|_{k}$ summability factors of infinite series, Tamkang J. Math. 16 (1985), 13-20.

[2] H. Bor, On two summability methods, Math. Proc. Cambridge Philos. Soc. 97 (1985), 147-149.

[3] H. Bor, A note on two summability methods, Proc. Amer. Math. Soc. 98 (1986), 81-84.

[4] H. Bor, A note on absolute summability methods, Real Anal. Exchange 18 (1992-1993), no. 1, 537-543.

[5] H. Bor and B. Thorpe, On some absolute summability methods, Analysis 7 (1987), 145-152.

[6] H. Bor and B. Thorpe, A note on two absolute summability methods, Analysis 12 (1992), 1-3.

[7] K. Chandrasekharan, The second theorem of consistency for absolutely summable series, Jour. Indian. Math. Soc. 6 (1942), 168-180.

[8] T. M. Flett, On an extension of absolute summability and some theorems of Littlewood and Paley, Proc. London Math. Soc. 7 (1957), 113-141.

[9] E. Kogbetliantz, Sur les séries absolument summables par la méthode des moyennes arithmétiques, Bull. Sci. Math. 49 (1925), no. 2, 234-256. 
[10] M. A. Sarigol, Necessary and sufficient conditions for the equivalence of the summability methods $\left|\bar{N}, p_{n}\right|_{k}$ and $|C, 1|_{k}$, Indian J. Pure Appl. Math. 22 (1991), 483-489.

[11] M. A. Sarigol, On the absolute Riesz summability factors of infinite series, Indian J. Pure Appl. Math. 23 (1992), 881-886.

[12] M. A. Sarigol, Characterization of summability factors for Riesz methods, J. Univ. Kuwait (science) 21 (1994), 1-6. 